The Royal College of Psychiatrists' Faculty of Medical Psychotherapy has recently been working towards an education strategy for the renewal and development of a more psychotherapeutic psychiatry, with the aim of bringing psychotherapy to the heart of psychiatry. I think that the model of meaning and mind that psychotherapy brings to the practice of psychiatry is crucial in enabling us to work with our disturbed patients, and as such it should be embedded into training.

1 Barras M, Harris J. Psychiatry recruited you, but will it retain you? Survey of trainees' opinions. Psychiatrist 2012; 36: 71-7.

2 Menzies-Lyth I. Containing Anxiety in Institutions. Free Association Books, 1988

3 Ballatt J, Campling P. Intelligent Kindness: Reforming the Culture of Healthcare. RCPsych Publications, 2011.

Meave T. Fingleton, locum consultant medical psychotherapist, The Pines Psychotherapy Service, Coventry and Warwickshire Partnership NHS Trust, Warwick, email: meave.fingleton@nhs.net

doi: $10.1192 / \mathrm{pb} .36 .5 .196 \mathrm{~b}$

\section{MRCPsych CASC exam: is there a better choice?}

The basic intention behind the final membership exams is to test the abilities and competencies of the candidates at the consultant level. An ideal exam offers equal chance and challenge to all its candidates. It checks both elementary and higher knowledge in all the necessary domains of the subject. A final membership exam should ideally be exhaustive, with an intrinsic ability to test the range, width and depth of the candidate's knowledge.

The Royal College of Psychiatrists' exams have been globally known for their excellence and high standards. MRCPsych Paper 1, 2 and 3 check the theoretical knowledge of the candidate, but owing to the complex nature of mental disorders, a psychiatrist needs to have a much wider and in-depth understanding rather than mere theoretical knowledge of the subject. The Clinical Assessment of Skills and Competencies (CASC) mainly appears to be good at testing the communication skills, mannerisms, body language and the ability of a candidate to handle a situation. This is clearly reflected in the high passing rates in CASC of graduates from the UK and other countries where English is the primary language compared with candidates from other countries. ${ }^{1}$ If we look at the passing rates of the practical components of the old MRCPsych Part 2 (i.e. individual patient assessment and patient management problems), we will see that the gap between the passing rates of UK graduates (including candidates from countries with English as their first language) and of the candidates from other countries is much narrower. ${ }^{2}$

To make matters worse for overseas candidates, there are so many variables in the present CASC exam that it is almost impossible for the candidates to understand where they are faltering. Some candidates seem unable to tell why they passed or why they failed. This has caused a lot of anxiety, discouragement and frustration, leading to a feeling of helplessness among overseas candidates who repeatedly fail at this exam despite scoring highly in their theory exams. On the other hand, CASC has its own advantages of being a fair deal to all examinees, covering various subspecialties at the same time, checking the ability of a candidate to handle a difficult clinical situation or for that matter the ability to control the interview. The local candidates seem to have an undue advantage when it comes to testing these skills. Being born and brought up in the UK (or in countries with English as the first language) gives them an upper hand when it comes to testing the doctor-patient interaction. For candidates whose primary language is not English, passing this exam seems to be comparatively a much more difficult task. In an attempt to 'perform a task in 7 minutes or 10 minutes in an artificial situation', the 'performer' just 'spits' out whatever he/she has crammed up, although in a sophisticated and palatable manner. There is no time to think, understand, plan or use any innovative strategies utilising the vibrantly balanced bio-psychosocial model embedded in the spirit of psychiatric management.

This is the final exam and we are testing the basic skills such as overdose, psychopathology and Mini-Mental State Examination (MMSE). The old-style MRCPsych exam Part 2 had great advantages. It presented the examinees with complex psychiatric situations (patient management problems) and offered them a chance to think, analyse, innovate and use a multidimensional biopsychosocial model when faced with questions directly from the consultants who had a much deeper knowledge in that field. It had the ability to analyse and evaluate the in-depth understanding of the system and the ways in which psychiatry works. The examinee's attitude, competence and excellence could then be gauged in the right way. Old-style exams carried a great advantage of being 'face to face' viva with consultants (rather than with an actor and consultant being a silent watcher as in (ASC) and provided a much more robust assessment of the quality and range of the candidate's knowledge at both academic and pragmatic levels. Few disadvantages of the old style examination were that it involved the real patient (individual patient assessment) who might have been slightly drowsy or restless due to side-effects of medications and it was very difficult to standardise as candidates often saw different patients.

There can never be an ideal exam and people will always have complaints, but if we combine the present CASC style with the old style of MRCPsych exam, we can have the best of both.

Considering the highly acclaimed quality of exams conducted by the UK medical Royal Colleges, it is time to reconsider and realise that the exam should not put any of the candidates at a disadvantage just because of their language and be equally challenging not only to people whose primary language is not English but also to people who have a limited knowledge of psychiatry. It would be a fair and balanced exam if instead of having 16 CASC stations there were 8 CASC stations and 8 stations of patient management problems/vivas so that it could provide candidates with a platform to prove their substance in both domains, namely communication and a thorough knowledge of the functioning of psychiatry as a system. Therefore, if two of the above are integrated, it may come closer to an ideal exam.

1 Bateman A. MRCPsych Examinations Cumulative Results 2008-2010. Royal College of Psychiatrists, 2011.

2 Oyebode F, Furlong E. MRCPsych examinations: cumulative results 1997-2002. Psychiatr Bull 2007; 31: 61-4.

Gursharan Kashyap, MBBS, DCH, MD, MRCPsych, ST4 trainee in general adult psychiatry, South Essex Partnership University NHS Foundation Trust (SEPT), email: gursharan.kashyap@sept.nhs.uk; Akeem Sule, MRCPsych, consultant psychiatrist, SEPT.

doi: 10.1192/pb.36.5.197 